\title{
Discovery of large scale shock fronts correlated with the radio halo and radio relic in the $\mathbf{A} 2163$ galaxy cluster ${ }^{\star}$
}

\author{
Sophia Thölken ${ }^{1}$, Thomas H. Reiprich ${ }^{1}$, Martin W. Sommer ${ }^{1}$, and Naomi Ota ${ }^{2}$ \\ 1 Argelander-Institut für Astronomie, Universität Bonn, Auf dem Hügel 71, 53121 Bonn, Germany \\ e-mail: thoelken@astro.uni-bonn.de \\ 2 Department of Physics, Nara Womens University, Kitauoyanishi-machi, Nara 630-8506, Japan
}

Received 8 August 2018 / Accepted 17 September 2018

\begin{abstract}
Context. Galaxy clusters form at the intersections of the filamentary large scale structure in merging events and by the accretion of matter along these filaments. Imprints of these formation processes should be visible in the intracluster medium and can arise in shock fronts, which are detectable via discontinuities in, for example, the gas temperature and density profiles. However, relatively few observational examples of prominent shocks have been detected in X-rays so far.

Aims. In this study, we investigate the X-ray properties of the intracluster gas and the radio morphology of the extraordinary cluster A2163. This cluster shows an irregular morphology in various wavelengths and has one of the most luminous and extended radio halos known. Additionally, it is one of the hottest clusters known. We aim to measure the temperature and density profiles in two azimuthal directions to search for the presence of shock fronts.

Methods. We performed a spectral analysis of data from two Suzaku observations, one in the north-east (NE) and one in the southwest (SW) direction of A2163, and used archival XMM-Newton data to remove point sources in the field of view. We deprojected the temperature and density profiles and accounted for the Suzaku point spread function. From the detected discontinuities in the density and temperature profiles, we estimated the Mach numbers and velocities of the shock fronts. To compare our findings in the X-ray regime with the radio emission, we obtained radio images of the cluster from an archival Very Large Array (VLA) observation at $20 \mathrm{~cm}$.

Results. We identify three shock fronts in A2163 in our spectral X-ray study. A clear shock front lies in the NE direction at a distance of $1.4 \mathrm{Mpc}$ from the center, with a Mach number of $M=1.7_{-0.2}^{+0.3}$, estimated from the temperature discontinuity. This shock coincides with the position of a known radio relic. We identify two additional shocks in the SW direction, one with $M=1.5_{-0.3}^{+0.5}$ at a distance of $0.7 \mathrm{Mpc}$, which is likely related to a cool core remnant, and a strong shock with $M=3.2_{-0.7}^{+0.6}$ at a distance of $1.3 \mathrm{Mpc}$, which also closely matches the radio contours. The complex structure of A2163 as well as the different Mach numbers and shock velocities suggest a merging scenario with two unequal merging constituents, where two shock fronts emerged at an early stage of the merger and traveled outwards while an additional shock front developed in front of the merging cluster cores.
\end{abstract}

Key words. galaxies: clusters: general - galaxies: clusters: individual: A2163 - X-rays: galaxies: clusters - radio continuum: general

\section{Introduction}

At the intersections of the filamentary large scale structure, the largest distinct building blocks in the universe, galaxy clusters, are located (e.g. Borgani \& Guzzo 2001; Springel et al. 2005; Vogelsberger et al. 2014). Merging events of galaxy clusters and groups are the most energetic processes in the universe and a significant fraction of the energy is dissipated into shock heating of the intracluster medium (ICM; e.g. Ha et al. 2018; Hong et al. 2015; McCarthy et al. 2007). These shock fronts are visible in X-ray observations of the ICM as discontinuities in the temperature, density, and surface brightness (SB) profiles. A prominent example of a distinct merger shock is the Bullet cluster (Markevitch et al. 2002) with a large Mach number of $M \sim 3$ (Springel \& Farrar 2007). Often, cold fronts are observed in mergers (see Ghizzardi et al. 2010 and references therein), caused by the cool core remnants of the merging constituents, while the actual shock front is located in front of the cold front, as is also the case for the Bullet cluster.

$\star$ The reduced Figs. 1 and 2 (FITS files) are only available at the CDS via anonymous ftp to cdsarc.u-strasbg. fr (130.79.128.5) or via http://cdsarc.u-strasbg.fr/viz-bin/qcat?J/A+A/619/A68
Until now, relatively few detailed X-ray studies of shocks in the ICM have been performed. For example, Simionescu et al. (2009) studied the merging cluster Hydra A using deep XMMNewton observations and found a large scale shock, visible in the SB profile and pressure map, with a Mach number of about 1.3. This is a typical value for X-ray detected shocks, which usually have $M \lesssim 3$ (Markevitch \& Vikhlinin 2007).

A recent study by Botteon et al. (2018) searched for discontinuities in a sample of 15 clusters with Chandra and found six shock fronts, eight cold fronts, and several discontinuities of uncertain origin. All the detected shocks have Mach numbers smaller than 2, estimated from the temperature and density profiles.

Another example, studied by Bourdin et al. (2013) with XMM-Newton, is the cluster A521. This cluster exhibits two cold fronts that separate the merging constituents and two shock fronts that propagate outwards in east and south-west directions. The shock heated regions and one of the shock fronts show spatial correlations with a radio halo and a radio relic, respectively. Radio halos are typically associated with disturbed or merging clusters, suggesting that the acceleration of electrons, responsible for the radio emission, is produced by turbulence in the ICM 
following a merger (e.g., Eckert et al. 2017; Brunetti \& Lazarian 2011, 2007). Other examples of shocks have, for example, been observed by Macario et al. (2011), Akamatsu et al. (2012, 2017), and Hattori et al. (2017), for the clusters A754, A3376, A2255, and A2744, respectively. In all cases, radio emission is observed and the $\mathrm{X}$-ray detected shocks seem to be related to radio relics. For A3376 and A2744, the authors report high Mach numbers of $M \sim 3$ and $M=3.7 \pm 0.4$, respectively, estimated from the gas temperature jump. A review about X-ray detected shocks in the ICM can be found in Markevitch \& Vikhlinin (2007).

Ogrean et al. (2014) detected multiple temperature and density discontinuities in the merging cluster CIZA J2242.8+ 5301 using Chandra and Suzaku data. This cluster hosts a double radio relic system and the radio-derived Mach number is almost a factor of two larger then the Mach number derived from the Suzaku X-ray temperature profile. This discrepancy between radio- and X-ray-derived Mach numbers is a known conundrum. Hong et al. (2015) analyzed cosmological hydrodynamic simulations, adopting the diffusive shock acceleration (DSA) model, and extracted radio and X-ray shock properties in the two-dimensional (2D) observer plane. They found that projection effects along the line of sight have a significant impact and radio observations tend to detect stronger shocks while $\mathrm{X}$-ray-derived Mach numbers are typically lower.

In this work, we study the disturbed cluster A2163 ( $z=$ 0.203, Struble \& Rood 1999), which is one of the hottest known clusters with a gas temperature of $T=14.6_{-0.8}^{+0.9} \mathrm{keV}$ (Elbaz et al. 1995) and also shows prominent radio emission (Feretti et al. 2001). We analyze two Suzaku observations in the north-east (NE) and south-west (SW) directions and investigate the ICM properties with respect to the presence of possible shock fronts.

Previous detailed studies of A2163 in the radio and optical band revealed a complex merging situation with several substructures and likely two constituents in the merging process. Okabe et al. (2011) performed a weak lensing analysis of A2163 and found a bimodel mass distribution. The mass distribution coincides with the galaxy density distribution but seems spatially offset from the brightest X-ray emitting regions, which the authors interpret in terms of ram pressure stripping of the gas in the merging process. Feretti et al. (2001) identified one of the largest known radio halos in A2163, which shows spatial correlations to the X-ray SB. Their radio analysis showed the presence of a radio structure in the NE direction, which was classified as a radio relic. Later, Feretti et al. (2004) investigated the spectral index map of A2163, which supported the interpretation that the previously identified radio feature is indeed a radio relic. Maurogordato et al. (2008) compared the findings in the radio regime with the galaxy density and found an even more pronounced correlation and several subclumps with clear galaxy overdensities.

An XMM-Newton and Chandra X-ray study of A2163 was performed by Bourdin et al. (2011). Their findings, obtained from spectral imaging, suggest that the ICM is in a highly disturbed state with a hotter region in the NE direction and a cool core remnant located SW of the cluster center. In their interpretation, the cool core was likely separated from its host halo. A galaxy overdensity close by Maurogordato et al. (2008) lends support to this interpretation. The cool core is likely to be moving westwards and also exhibits a cold front as found by Bourdin et al. (2011) using Chandra data. The authors also identified a prominent structure north of the cluster center, which we also see in our Suzaku observation (see Fig. 1), however, they state that no indications for an interaction with the main cluster are found.

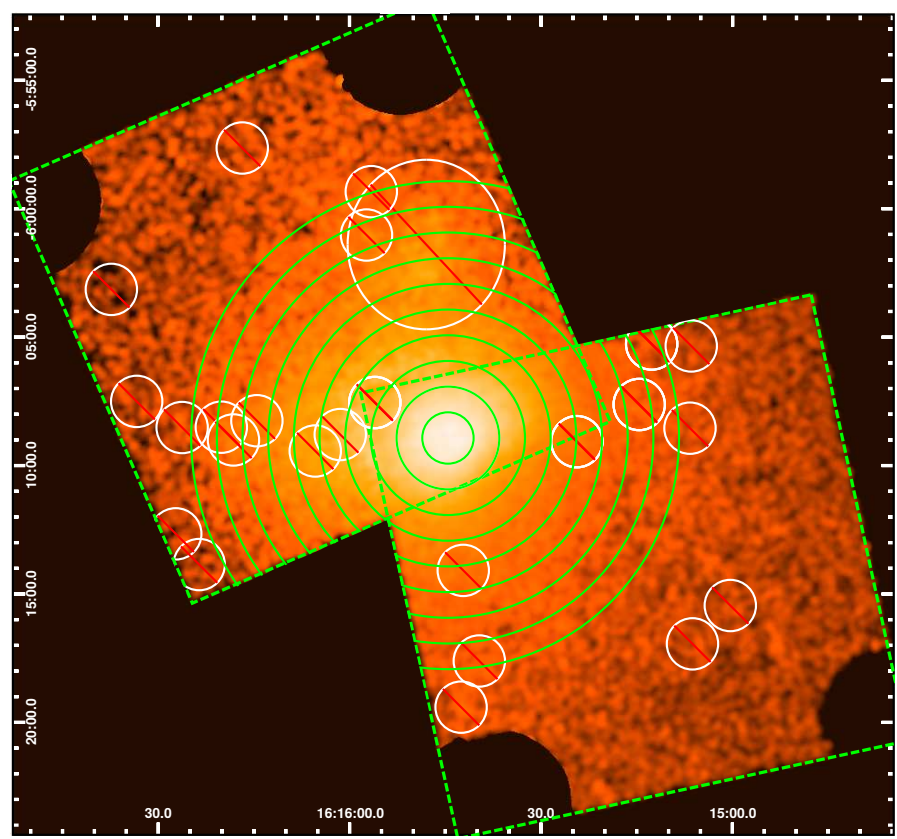

Fig. 1. Suzaku pointings (green dashed boxes), extraction regions for the Suzaku analysis (green annuli), and removed (point source) regions (white circles). Point sources are removed above a flux limit of $3 \times 10^{-14} \mathrm{erg} \mathrm{s}^{-1} \mathrm{~cm}^{-2}$. As in Bourdin et al. (2011), the structure in the north is removed because it seems unrelated to the main cluster.

Ota et al. (2014) looked for hard X-ray emission in A2163 using Suzaku Hard X-ray Detector (HXD) data. They find significant emission in the $12-60 \mathrm{keV}$ band and a multitemperature structure with a high temperature component in the NE direction, but no significant non-thermal X-ray emission. A detailed comparison of our work to these findings and possible interpretations is given in Sect. 5.

The extensive multiwavelength studies of A2163 brought up interesting results. However, despite its disturbed appearance, no shock fronts have been detected so far, although their presence seems likely given the cluster's substructured morphology. With our Suzaku analysis, we aim to identify these shocks and we are able to robustly probe the gas properties out to larger radii than is possible with XMM-Newton or Chandra due to Suzaku's low and stable instrumental background.

Throughout the analysis we assume $\Omega_{\Lambda}=0.7, \Omega_{\mathrm{m}}=$ $0.3, H_{0}=70 \mathrm{~km} \mathrm{~s}^{-1} \mathrm{Mpc}^{-1}$, which corresponds to a scale of $3.3 \mathrm{kpc} /{ }^{\prime \prime}$ at the cluster redshift of $z=0.203$. All uncertainties are quoted at the $68 \%$ level.

\section{Observations and data reduction}

\subsection{Radio data}

The radio halo in A2163 was first studied by Feretti et al. (2001), using the same Very Large Array (VLA) data that we use here. A2163 was observed at $20 \mathrm{~cm}$ wavelength with the VLA C and D configurations under project number AF328 in 1998 and 1999 , using central frequencies of $1.36 \mathrm{GHz}$ and $1.46 \mathrm{GHz}$ with $25 \mathrm{MHz}$ bandwidth per frequency in the $\mathrm{C}$ configuration and $50 \mathrm{MHz}$ bandwidth per frequency in the D configuration. The on-source integration time was $3.9 \mathrm{~h}$ (C configuration) and $3.4 \mathrm{~h}$ (D configuration). 
Table 1. Details of the Suzaku observations of A2163.

\begin{tabular}{lccccc}
\hline \hline & Date & Pointing (RA, Dec) & Exp. Time & Obs-ID & PI \\
\hline South-west (SW) & 2008 Aug & $(243.8071,-6.2200)$ & $109.2 \mathrm{ks}$ & 803071010 & T. H. Reiprich \\
North-east (NE) & 2009 Feb & $(244.0216,-6.0449)$ & $38.6 \mathrm{ks}$ & 803022010 & N. Ota \\
\hline
\end{tabular}

Notes. The exposure time is the cleaned exposure time after data reduction.

\subsection{X-ray data}

In this work, we analyze two Suzaku XIS observations of A2163 in a NE and SW direction of $39 \mathrm{ks}$ and $109 \mathrm{ks}$ cleaned exposure time, respectively. Details of the observations can be found in Table 1. Additionally, we used archival XMM-Newton data (PI: H. Bourdin, Obs.-ID: 069450010) to detect point sources in the field of view. We followed the standard Suzaku data reduction procedure, which includes the following steps: assign coordinates, time, and pixel quality flags to each event, apply gain- and charge-transfer-inefficiency correction, and identify anomalous pixels. For the geomagnetic cut-off rigidity, we use a limit of COR $2>6$ and events falling in the second trailing rows of the charge injection rows are discarded.

Suzaku observations can be contaminated by solar flares, which cause solar-wind-charge-exchange emission. We performed a flare filtering by applying a three-sigma clipping to the light curves and removing the corresponding time intervals. Point sources were detected using the archival XMM-Newton observation, which we also cleaned for flares and which covered the analyzed area in both Suzaku pointings. The task emldetect was used to detect point sources in the field of view in two energy bands from $0.5-4.5 \mathrm{keV}$ and $4.5-12.0 \mathrm{keV}$. We applied the energy conversion factors from Rosen et al. (2016). Not all of the detected sources could be removed from the Suzaku observation as this would remove a significant amount of area due to Suzaku's larger point spread function (PSF). Thus, we chose a flux limit of $3 \times 10^{-14} \mathrm{erg} \mathrm{s}^{-1} \mathrm{~cm}^{-2}$. In the NE direction, we identify the same substructure as found by Bourdin et al. (2011), which we exclude from the analysis. All removed areas are shown in Fig. 1.

\section{Analysis}

\subsection{Radio analysis}

The interferometric radio data were calibrated using the Common Astronomy Software Applications (McMullin et al. 2007) package. Primary flux calibration was performed against the known calibrator 3C286, adopting the flux scale of Perley \& Butler (2013). Phase calibration was carried out against bright nearby compact sources (that were found to be observed approximately every thirty minutes) for which we in turn determined gain solutions from the primary calibrator.

We performed several iterations of self-calibration for each antenna configuration separately, to improve upon the phase solutions. Upon combining the data from the two configurations, we performed one iteration of amplitude self-calibration, with solutions averaged over one hour, followed by one final iteration of phase self-calibration. Residual phase errors were found to be less than a few degrees across all antennae.

To image the radio halo, we first made a model image of the compact sources in the field, using only baselines longer than $1.8 \mathrm{k} \lambda$ (corresponding to around $380 \mathrm{~m}$ at $20 \mathrm{~cm}$ wavelength) and multifrequency deconvolution (Rau \& Cornwell 2011) with two Taylor terms to model spectral slopes in the continuum. De-gridding the compact source model to $u v$ space, we sub- tracted the model from the visibilities and proceeded to image the radio halo using the short interferometer spacings, which are sensitive to the extended emission. To image the extended emission, we used Briggs weighting with robust $=1$ and a $u v$ taper at $45^{\prime}$. Due to the large size of the radio halo, we used multiscale CLEANing to deconvolve the image. Our final image (Fig. 2) has a reconstructed circular Gaussian beam of $50^{\prime \prime} \times 50^{\prime \prime}$ at fullwidth half-maximum (FWHM). Structure is recovered to scales of approximately $12^{\prime}$, which is close to the limit of what can be imaged with the VLA.

\subsection{X-ray analysis}

\subsubsection{Background}

The Suzaku non-X-ray background (NXB) is caused by highly energetic particles hitting the detector and producing continuum and fluorescent line emission. This background is estimated from night earth observations in a time interval of \pm 150 days around the observation date and subtracted from the source spectra. For the creation of the NXB spectra, we followed the improved treatment of the flickering pixels ${ }^{1}$.

The X-ray background is mainly composed of three components: a local component from the local hot bubble and solar wind charge exchange (this component is called LHB in the following), a milky way halo component, and the superposition of distant active galactic nuclei (AGN) causing a diffuse background (called CXB in the following). We model these components using an apec and absorbed apec model for the LHB and the halo component, respectively, and an absorbed power law for the CXB component. The LHB temperature is fixed to $0.1 \mathrm{keV}$ and the CXB component has a photon index of 1.41 (De Luca \& Molendi 2004). The redshift of the apec models is fixed to 0 and the abundance is set to 1 . The temperature of the halo component is fixed to $0.28 \mathrm{keV}$ (e.g., Hoshino et al. 2010; Akamatsu et al. 2011). In addition to the Suzaku data, we use ROSAT All-Sky Survey data to constrain the background model parameters in a region far off the center where no cluster emission is expected ${ }^{2}$. We created ancillary response files (ARF) for the background spectra using xissimarfgen and assuming a uniform distribution.

\subsubsection{PSF correction}

The limited spatial resolution of Suzaku makes a correction for the effect of the PSF necessary, which is $1.8^{\prime}$ half-power diameter. Therefore, we simulated the mixing of photons between the different annuli for each of the observations separately with xissim. As in Thölken et al. (2016), we used an iterative approach for the input image of the simulation. First, we obtained background-subtracted SB profiles from Suzaku images

\footnotetext{
1 See heasarc.gsfc.nasa.gov/docs/suzaku/analysis/ xisnxbnew.html.

2 Spectra were extracted with the HEASARC X-ray background tool
} heasarc.gsfc.nasa.gov/cgi-bin/Tools/xraybg/xraybg.pl. 


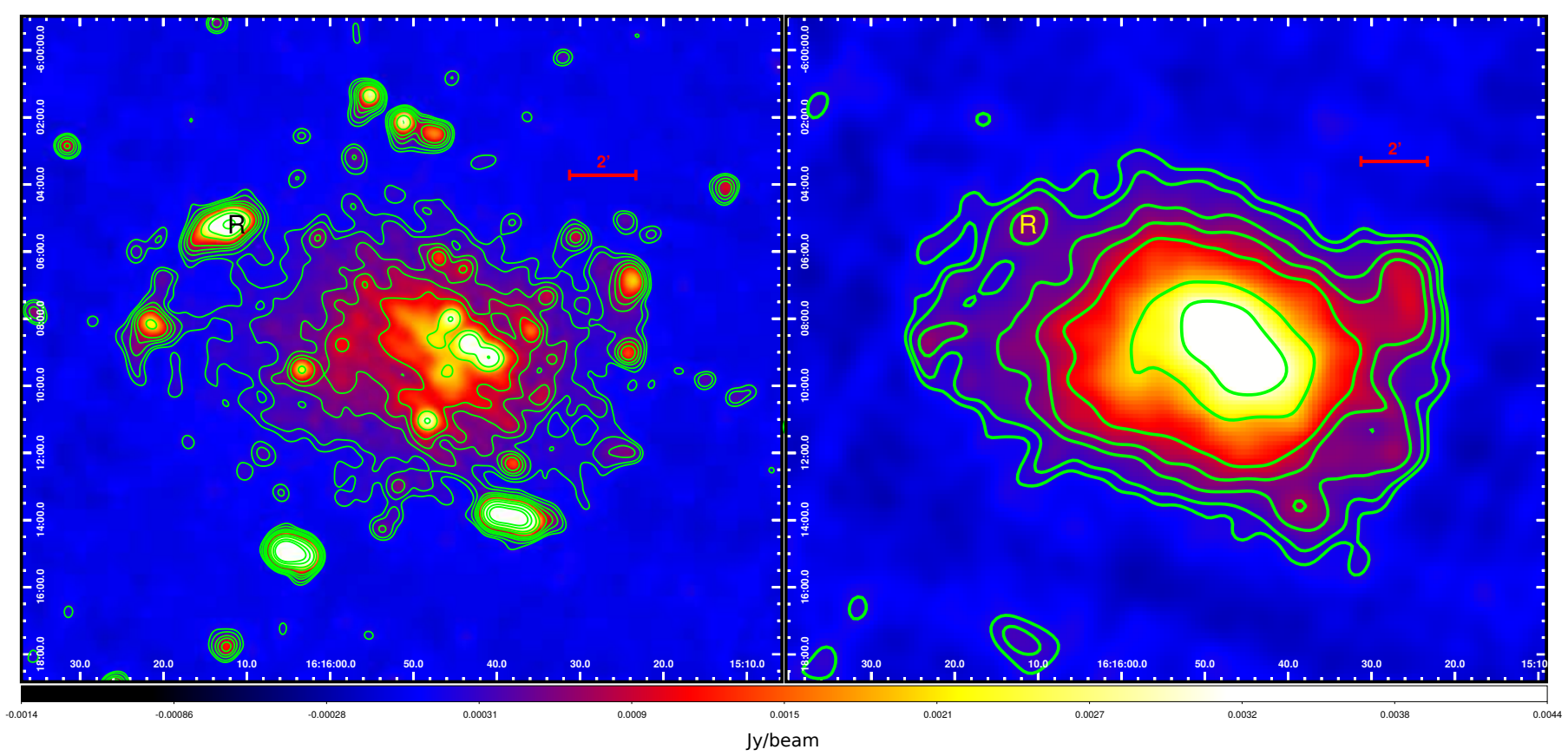

Fig. 2. Radio images of A2163 at $20 \mathrm{~cm}$. The contour levels are $0.2,0.3,0.5,0.7,1,2,3,5,7 \mathrm{mJy}_{\text {beam }}{ }^{-1}$. The letter "R" indicates the position of the radio relic, as identified by Feretti et al. (2001). Left: radio map with a circular Gaussian beam size of $30^{\prime \prime}$ FWHM. The noise level is $0.05 \mathrm{mJy}_{\text {beam }}{ }^{-1}$. Right: image of the extended emission after the removal of compact sources with a circular reconstructed beam size of $50^{\prime \prime}$ FWHM. The noise level is $0.1 \mathrm{mJy}^{\text {beam }}{ }^{-1}$.

in the NE and SW directions, which we fit by single-beta models and used these models as inputs to the simulation. From this simulation, we obtained PSF-correction factors that are introduced into the fitting procedure. From this spectral fit, PSF-corrected SB profiles were obtained, which were then again used as inputs to the simulation and the analysis was repeated. Details of this procedure can be found in Thölken et al. (2016). To reduce the correlations in the fit due to the PSF correction, we accounted for PSF mixing factors $\geq 10 \%$ as we do not expect strong influence from annuli with lower mixing factors. However, significant correlations between neighboring annuli were still present, which were found to result in temperature "oscillations" in the fit. We thus regularized the temperature and abundance values by allowing them to vary within $\pm 2 \sigma$ of the values of a PSFuncorrected fit. However, the normalization was not constrained and we discuss the effect of the regularization on our results in Sect. 4.3.

\subsubsection{Fitting procedure}

We analyzed the observations in the NE and SW directions separately and extracted spectra in ten and nine radial bins, respectively, each with $1^{\prime}$ width. We examined the signal-tobackground ratio $\left(N_{\text {source }} / N_{\text {bkg }}\right)$ in each annulus and limited the number of regions such that the ratio is approximately one in the outermost annulus. The extraction regions are shown in Fig. 1.

The source emission is modeled by an absorbed apec model with a fixed redshift of $z=0.203$ and the column density taken from Willingale et al. (2013), which includes molecular hydrogen. Since A2163 lies behind a relatively dense hydrogen cloud, we also tested the impact on the temperature profile when leaving the column density in each spectral annulus as a free fit parameter, but found only marginal differences in each bin.

The abundance table from Asplund et al. (2009) is used throughout this analysis. The metal abundance is estimated in four radial bins, that is, this parameter is linked across several annuli according to Tables A.1 and A.2, for the NE and SW directions, respectively.

Ancillary response files for both observations were created using the same iterative approach for the beta-model profiles as used for the PSF simulation in Sect. 3.2.2. All spectra were grouped in at least 25 counts per bin and analyzed in the energy range $0.7-10 \mathrm{keV}$, except for the outermost three (two) annuli in the NE (SW) direction, for which the fitting range is $0.7-7 \mathrm{keV}$, to achieve a sufficient signal-to-background ratio.

\subsubsection{Deprojection}

To deproject our results, we followed the method described in Thölken et al. (2016), based on Ettori et al. (2002), and assumed piecewise functions to model the electron density and temperature profiles with the discontinuities caused by the shock fronts. The temperature and density profiles were deprojected simultaneously and correlations between the data points due to the PSF correction were taken into account using the covariance matrix from the spectral fit. The irregular morphology of the cluster makes it necessary to treat the NE and SW direction separately as described in the following.

The temperature model in the NE direction is described by a simple step function

$T^{\mathrm{NE}}(R)=\left\{\begin{array}{ll}T_{0}^{\mathrm{NE}}, & R \leq R_{\mathrm{j}} \\ T_{1}^{\mathrm{NE}}, & R>R_{\mathrm{j}}\end{array}\right.$,

with $R_{j}$ being the position of the discontinuity. The electron density in the NE direction is, correspondingly, modeled by a piecewise power law

$n_{\mathrm{e}}^{\mathrm{NE}}(R)=\left\{\begin{array}{ll}n_{1} R^{-\eta_{1}}, & R \leq R_{j} \\ n_{2} R^{-\eta_{2}}, & R>R_{j}\end{array}\right.$,

with normalizations $n_{1}$ and $n_{2}$, and slope parameters $\eta_{1}$ and $\eta_{2}$. This simplified density model is not able to reproduce the central 


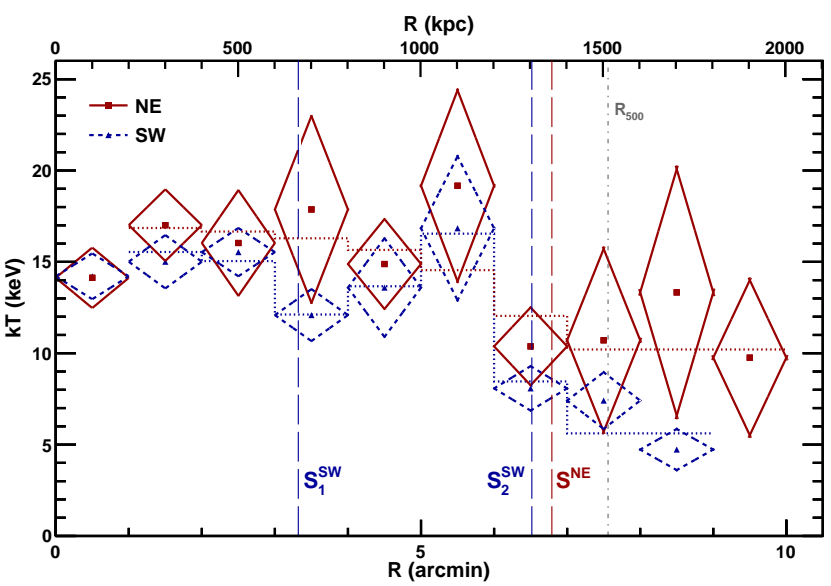

Fig. 3. Projected, PSF-corrected Suzaku temperature profiles of A2163 obtained in NE (solid red diamonds) and SW (dashed blue diamonds) direction. The vertical dashed lines show the jump positions of the detected shocks as obtained from our deprojection procedure while dotted lines correspond to the projected best-fit temperature models (see Sect. 3.2.4 for details about the deprojection). The gray vertical dotdashed line indicates $R_{500}$, as obtained by Radovich et al. (2008) from a weak-lensing analysis.

part of the cluster for which usually a (double) beta-model is needed. Thus, we exclude the central bin in the deprojection.

In the SW direction, a more complex modeling is necessary. The SW temperature profile is discussed in detail in Sect. 4 and we anticipate the results here to argue for the deprojection models that we used. In a first test, we applied the same temperature and density models for the deprojection as used in the NE direction. However, with these models, we find a bad description of our measured quantities, $\left(\chi^{2} /\right.$ d.o.f $\left.=3.6\right)$, which seem to require more advanced deprojection models. Nevertheless, this first test with the simple single-jump models indicates that a shock position around $\sim 3.5^{\prime}$ is preferred instead of the clearly visible jump in the SW temperature profile at around 6' (see Sect. 4, Fig. 3). For this reason, we tested models that allow for two discontinuities at the positions $R_{\mathrm{j}, 1}<R_{\mathrm{j}, 2}$. In the temperature profile, the first and second position are smoothly connected via a Sigmoid function. These models yield a better description of our measured values in the SW direction, regarding the reduced Chi-squared $\left(\chi^{2} /\right.$ d.o.f $\left.=0.8\right)$ and are therefore used in the following. Using the F-test, the single-jump model is rejected with a false-rejection probability of less than $10 \%$. Thus, the final SW temperature model is given by

$T^{\mathrm{SW}}(R)=\left\{\begin{array}{ll}T_{0}^{\mathrm{SW}}, & R \leq R_{\mathrm{j}, 1} \\ S(R), & R_{\mathrm{j}, 1}<R \leq R_{\mathrm{j}, 2} \\ T_{2}^{\mathrm{SW}}, & R>R_{\mathrm{j}, 2}\end{array}\right.$,

with the Sigmoid function

$$
S(R)=\frac{\left(T_{1, b}-T_{1, a}\right)}{1+\exp \left(-m\left[R-0.5\left(R_{\mathrm{j}, 2}-R_{\mathrm{j}, 1}\right)\right]\right)}+T_{1, a},
$$

which models the smooth transition between the two temperatures at the jump positions $T_{1, a}<T_{1, b}$ with slope $m$. The turning point is chosen to lie at halfway between the two jumps.

The density is, correspondingly, described by

$$
n_{\mathrm{e}}^{\mathrm{SW}}(R)= \begin{cases}n_{0}^{\mathrm{SW}} R^{-\alpha}, & R \leq R_{\mathrm{j}, 1} \\ n_{1}^{\mathrm{SW}} R^{-\beta}, & R_{\mathrm{j}, 1}<R \leq R_{\mathrm{j}, 2} \\ n_{2}^{\mathrm{SW}} R^{-\gamma}, & R>R_{\mathrm{j}, 2}\end{cases}
$$

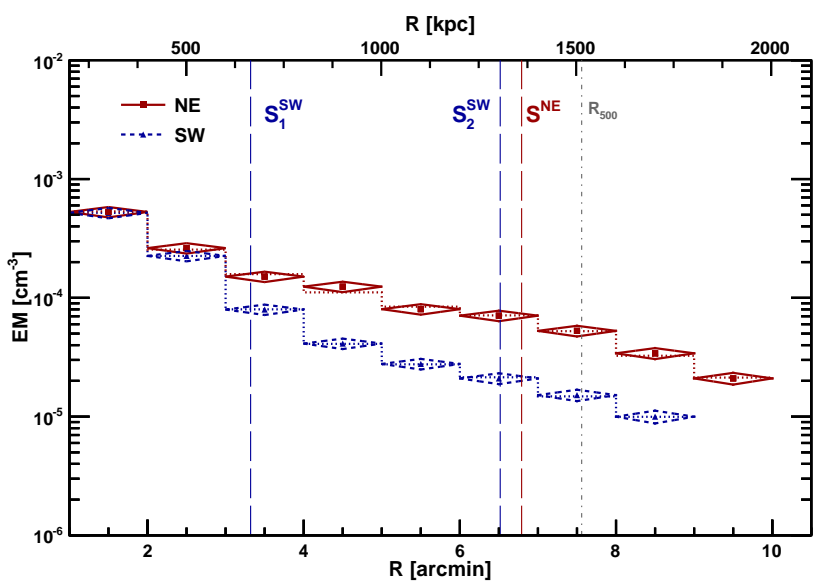

Fig. 4. Projected, PSF-corrected emission measure profile of A2163 obtained from the spectral fit for the NE (solid red diamonds) and SW (dashed blue diamonds) directions. Dashed and dotted lines have the same meaning as in Fig. 3. The innermost annulus is not shown as it is not considered in the deprojection procedure (see text). The profiles reproduced from the deprojection models (dotted lines) well match the measured profiles.

\section{Results}

\subsection{Radio emission}

Figure 2 shows the radio images obtained from our VLA analysis. As identified previously by Feretti et al. (2001), A2163 exhibits several compact sources, a large extended radio halo, and a radio relic in the NE. The latter is supported by measurements of the spectral index map between 0.3 and $1.4 \mathrm{GHz}$ by Feretti et al. (2004). The radio emission is slightly elongated in E-W direction, which supports the interpretation by Bourdin et al. (2011) and Maurogordato et al. (2008) that the merging is proceeding along this direction. A detailed comparison of the radio and $\mathrm{X}$-ray results is presented in Sect. 5.

\subsection{X-ray temperature and emission measure profiles}

Figure 3 shows the measured, PSF-corrected temperature profiles of A2163 in the NE and SW directions, as well as the projected model temperature values, which are obtained by projecting the best-fit model of the deprojection procedure along the line of sight. The projected models are able to well reproduce the observed profiles. The obtained temperatures are given in Tables A.1 and A.2 for the NE and SW directions, respectively. As expected, the profiles agree in the inner cluster region, where the two Suzaku pointings overlap, but show clear deviations farther out. In general, the temperature in the SW direction is lower compared to the NE direction, which has a relatively flat temperature profile. Most notably, both profiles exhibit a clear temperature drop around $6.5^{\prime}$, which was also seen in an earlier study by Markevitch \& Vikhlinin (2001) using a short Chandra observation, albeit with large uncertainties due to the short exposure.

In the SW direction, we measure an increase in temperature between the fourth and sixth radial bin, followed by a sudden drop from $\sim 17 \mathrm{keV}$ to $\sim 8 \mathrm{keV}$, which is a clear indication of a shock front. With our deprojection algorithm, we identify a second discontinuity located at $\sim 3.3^{\prime}$. By eye, an indication for this discontinuity in the measured temperature profile can be seen in the fourth annulus, where the temperature slightly drops. Also the emission measure profile, shown in Fig. 4, 
Table 2. Properties of the shock in NE and the two shocks in SW direction of A2163.

\begin{tabular}{|c|c|c|c|c|c|c|c|c|}
\hline & \multirow[b]{2}{*}{$R_{\mathrm{j}}(\operatorname{arcmin})$} & \multirow[b]{2}{*}{$R_{\mathrm{j}}(\mathrm{kpc})$} & \multicolumn{3}{|c|}{ Density estimates } & \multicolumn{3}{|c|}{ Temperature estimates } \\
\hline & & & $A$ & M & $v\left(10^{3} \mathrm{~km} \mathrm{~s}^{-1}\right)$ & $A$ & $M$ & $v\left(10^{3} \mathrm{~km} \mathrm{~s}^{-1}\right)$ \\
\hline$S_{1}^{\mathrm{SW}}$ & $3.3_{-0.3}^{+0.3}$ & $665_{-60}^{+55}$ & $1.5_{-0.3}^{+0.3}$ & $1.3_{-0.2}^{+0.2}$ & $2.1_{-0.3}^{+0.4}$ & $1.7_{-0.5}^{+1.3}$ & $1.6_{-0.5}^{+0.9}$ & $2.6_{-0.7}^{+1.5}$ \\
\hline$S_{2}^{\text {SW }}$ & $6.5_{-0.2}^{+0.3}$ & $1305_{-47}^{+68}$ & $0.8_{-0.1}^{+0.2}$ & - & - & $4.1_{-1.2}^{+1.4}$ & $3.2_{-0.7}^{+0.6}$ & $4.0_{-0.8}^{+0.8}$ \\
\hline$S^{\mathrm{NE}}$ & $6.8_{-0.4}^{+0.4}$ & $1360_{-78}^{+81}$ & $0.8_{-0.1}^{+0.1}$ & - & - & $1.7_{-0.2}^{+0.3}$ & $1.7_{-0.2}^{+0.3}$ & $2.7_{-0.4}^{+0.5}$ \\
\hline
\end{tabular}

Notes. The jump amplitude $A$ is the ratio of the respective quantity in front of and behind the shock, $M$ is the Mach number, and $v$ is the shock velocity. Where the jump amplitude is smaller than one, no Mach number and velocity estimates are given.
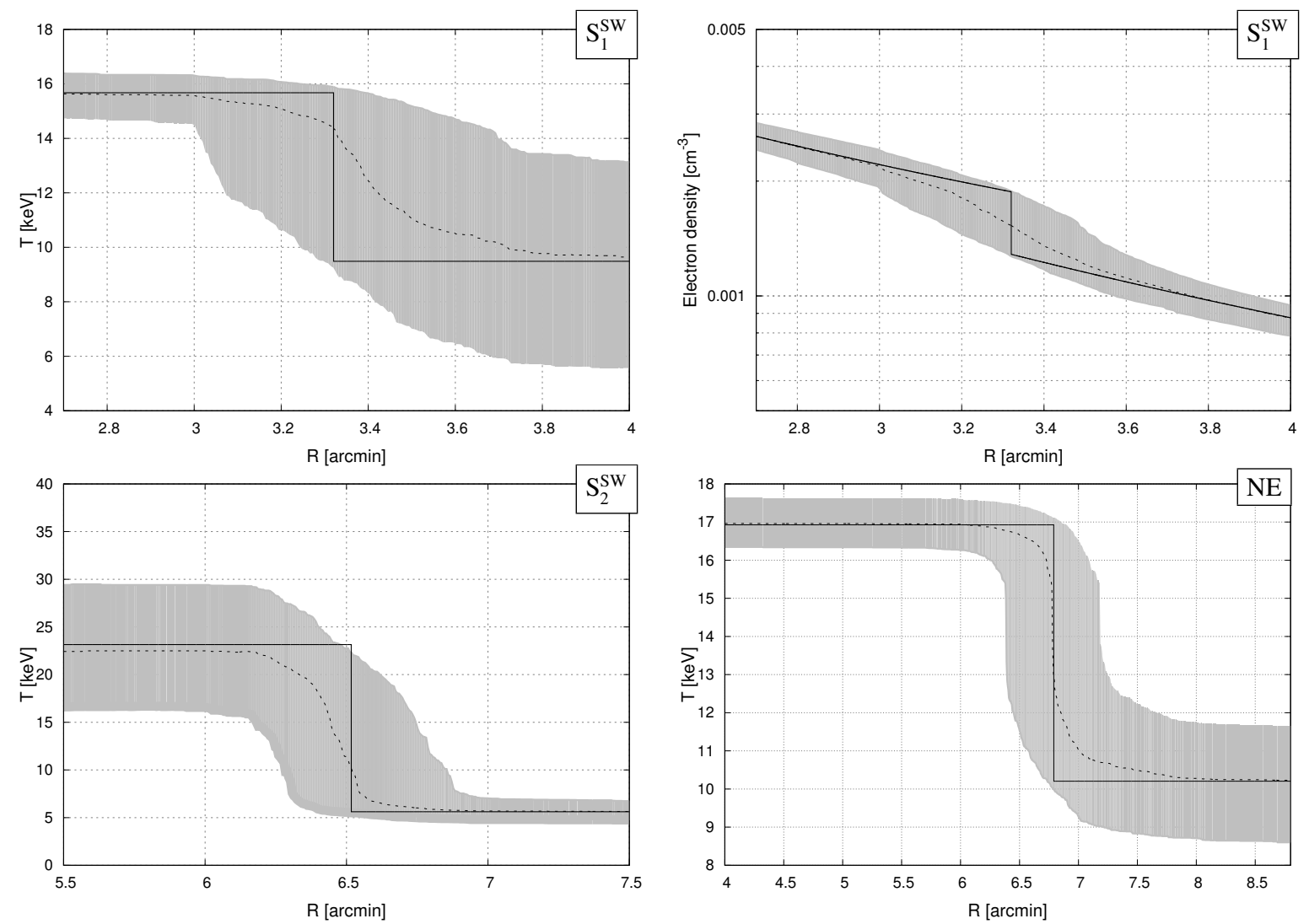

Fig. 5. Deprojected temperature and electron density profiles around the shock regions for the NE and SW directions. Solid lines correspond to the deprojected profiles, and gray shaded areas show the $68 \%$ confidence intervals estimated from 1000 Monte-Carlo realizations. The dashed lines show the median of all realizations. For details of the shock properties see Table 2. Top row: deprojected temperature (left) and density (right) profile around the jump position for the inner shock in the SW direction. Bottom left: deprojected temperature profile in the shock region for the outer shock in the SW direction. The profile slightly exceeds the $68 \%$ interval at the jump position because of the sharp discontinuity, which is smeared out in the MC realizations. Bottom right: deprojected temperature profile in the NE direction around the shock position.

shows a stronger drop around that radius than the NE direction and is overall clearly flatter than the SW profile. No edges are detected in the emission measure profile around the positions of the outer shocks. We therefore also checked the SB profile of the XMM-Newton observation but found no associated features, which is somewhat surprising given the clear temperature discontinuities. This has also been seen before, for example, by Akamatsu et al. (2017) who also could not identify an SB edge for their detected shock front despite a clear temperature jump across a radio relic. Interestingly, the SB profile of A2163 of an early ROSAT study (Elbaz et al. 1995) shows a slight hint of a feature around the outer shock positions, which is, however, not seen in our analysis, and deeper XMM-Newton or Chandra data might be required to further investigate this point. In the future, eROSITA (extended Roentgen Survey with an Imaging Telescope Array) might also help to resolve this issue with its very good soft spectral response (Merloni et al. 2012). In general, due to the strong gradient in the emission measure profiles, jumps caused by shock fronts might not be as clearly visible as the temperature discontinuities, especially at large radii.

Overall, we find that our measured profiles are very well reproduced by our deprojection method when projecting the best-fit deprojected models along the line of sight. The reduced $\chi^{2}$ values in the NE and SW directions are $\chi^{2} /$ d.o.f. $=0.3$ $\left(\chi^{2}=3.4\right)$ and $\chi^{2} /$ d.o.f. $=0.8\left(\chi^{2}=2.3\right)$, respectively. The low value in the NE direction (despite the simple deprojection model) is an indication that the uncertainties are likely to be 
Table 3. Properties of the shock in NE and the two shocks in SW direction without PSF-correction and temperature regularization.

\begin{tabular}{|c|c|c|c|c|c|c|c|c|}
\hline & \multirow[b]{2}{*}{$R_{\mathrm{j}}(\operatorname{arcmin})$} & \multirow[b]{2}{*}{$R_{\mathrm{j}}(\mathrm{kpc})$} & \multicolumn{3}{|c|}{ Density estimates } & \multicolumn{3}{|c|}{ Temperature estimates } \\
\hline & & & $A$ & $M$ & $v\left(10^{3} \mathrm{~km} \mathrm{~s}^{-1}\right)$ & $A$ & $M$ & $v\left(10^{3} \mathrm{~km} \mathrm{~s}^{-1}\right)$ \\
\hline$S_{1}^{\mathrm{SW}}$ & $3.4_{-0.3}^{+0.3}$ & $680_{-53}^{+63}$ & $1.2_{-0.3}^{+0.3}$ & $1.2_{-0.2}^{+0.2}$ & $2.1_{-0.3}^{+0.4}$ & $1.3_{-0.2}^{+0.3}$ & $1.3_{-0.2}^{+0.3}$ & $2.3_{-0.3}^{+0.5}$ \\
\hline$S_{2}^{\text {SW }}$ & $6.6_{-0.2}^{+0.3}$ & $1325_{-46}^{+60}$ & $1.0_{-0.2}^{+0.3}$ & - & - & $2.9_{-0.5}^{+0.6}$ & $2.5_{-0.3}^{+0.4}$ & $3.3_{-0.5}^{+0.5}$ \\
\hline$S^{\mathrm{NE}}$ & $6.6_{-0.4}^{+0.5}$ & $1330_{-72}^{+92}$ & $0.9_{-0.1}^{+0.1}$ & - & - & $1.4_{-0.2}^{+0.3}$ & $1.5_{-0.2}^{+0.3}$ & $2.6_{-0.3}^{+0.4}$ \\
\hline
\end{tabular}

Notes. Parameters are the same as in Table 2.

overestimated by the fit, caused by the correlations introduced by the PSF correction.

We quantify the temperature and density jumps and the resulting shock properties in the following section and give possible interpretations of the merging scenario in Sect. 5 .

\subsection{Properties of the shock fronts}

From the deprojected models, introduced in Sect. 3.2.4, we estimate the jump amplitudes (i.e., the ratio of the respective quantity in front of and behind the shock) in the temperature and (where possible) density profiles. From these values, the Mach numbers of the shocks are estimated using the Rankine Hugoniot jump conditions. The results are given in Table 2, and Fig. 5 shows the corresponding discontinuities in the deprojected profiles. The uncertainties are estimated from 1000 Monte-Carlo realizations of the measured profiles, for which we repeat the deprojection but reject unphysical realizations (e.g., negative temperatures). We include a $10 \%$ systematic error on the spectral normalizations, which comes from the uncertainty on the shape of the beta-model profile used in the ARF generation.

The inner shock in the SW direction (labeled $S_{1}^{\mathrm{SW}}$ ) is located at $3.3^{\prime}$ and the temperature and density profile yield consistent Mach numbers of $1.6_{-0.5}^{+0.9}$ and $1.3_{-0.2}^{+0.2}$, respectively, albeit with relatively large uncertainties. These Mach numbers correspond to shock velocities of $2100-2600 \mathrm{~km} \mathrm{~s}^{-1}$ and are typical values for X-ray detected shocks. The second shock in the SW direction $\left(S_{2}^{\mathrm{SW}}\right)$ as well as the shock in the NE direction $\left(S^{\mathrm{NE}}\right)$ are only detectable in the temperature profiles, whereas no clear discontinuities are found in the density profiles. The probable reasons for this are, on the one hand, Suzaku's large PSF and, on the other hand, the steep gradient of the density profile, which makes it more difficult to detect discontinuities.

The Mach number of the NE shock, estimated from the temperature jump, is $M=1.7_{-0.2}^{+0.3}$. The Mach number of the outer shock in the SW direction is $M=3.2_{-0.7}^{+0.6}$, which is among the strongest detected X-ray shocks and comparable to the shock front detected in the Bullet cluster. However, the uncertainties are relatively large and consequently also the shock velocity has a large range of $3200-4800 \mathrm{~km} \mathrm{~s}^{-1}$. The challenge of high collision velocities larger than $\sim 4000 \mathrm{~km} \mathrm{~s}^{-1}$ to the $\Lambda \mathrm{CDM}$ cosmology has been extensively discussed for the Bullet cluster (e.g., Hayashi \& White 2006; Springel \& Farrar 2007; Angus \& McGaugh 2008; Lee \& Komatsu 2010). However, we do not consider such a discussion meaningful here for A2163, given the large uncertainties on the Mach numbers.

As mentioned in Sect. 3.2.2, we regularized the temperature in the fitting procedure due to strong correlations caused by the PSF correction. We tested the implications of this procedure for the shock properties by performing the analysis without PSF correction, that is, without regularization.

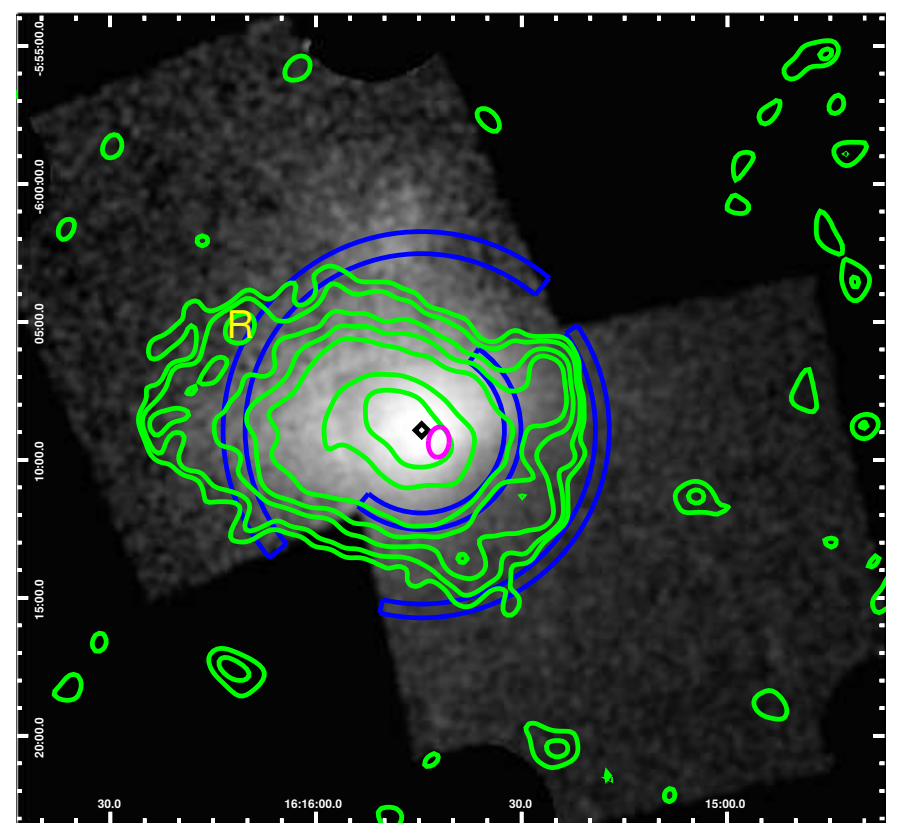

Fig. 6. Positions of the shock fronts in A2163 (blue), the X-ray emission peak (black diamond), and the approximate position of the cool core (magenta ellipse) found by Bourdin et al. (2011). The widths of the shock positions correspond to the uncertainties on the jump radius, estimated from the deprojection (see Table 2). Green contours show the extended radio emission with the same contour levels as in the right panel of Fig. 2. The yellow letter " $R$ " marks the position of the radio relic as identified in Feretti et al. (2001).

The values of this procedure are given in Table 3. The results agree well with the nominal values in Table 2 within the uncertainties. The jump amplitude (and corresponding Mach number) for the strong shock $S_{2}^{\mathrm{SW}}$ in the SW direction is slightly lower, which is not surprising because the effect of the PSF smears out temperature differences in neighboring annuli. However, these values are also consistent with the regularized PSF-corrected values within the uncertainties and we thus conclude that this method does not bias our estimated shock properties.

\section{Discussion and conclusions}

Figure 6 shows the radio contours of A2163 as well as the estimated shock positions from this analysis. Overall, we see a tight correlation between the X-ray detected shock positions and the radio contours. A general correlation between the radio and X-ray features of A2163 was also previously observed by Feretti et al. (2001) but, so far, has not been associated with shock fronts. 


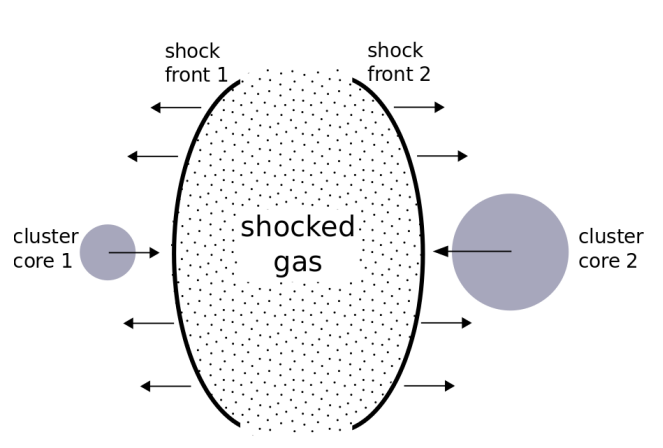

early merger stage

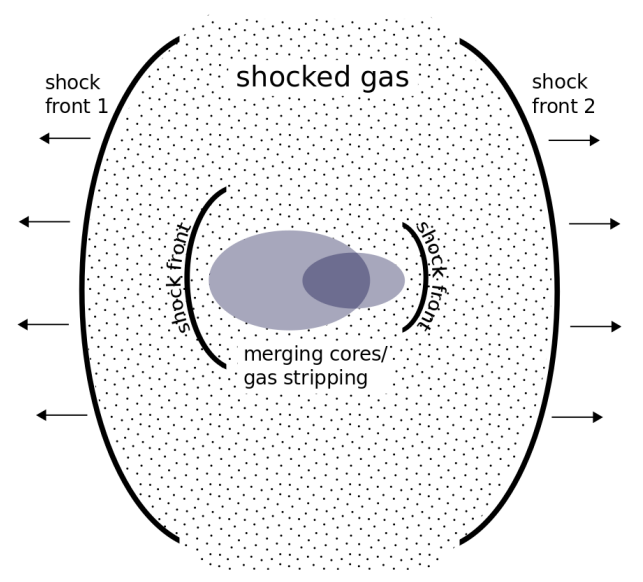

later merger stage
Fig. 7. Possible merger scenario adapted from Markevitch et al. (2000). At an early stage of the merger (left), the gas between the merging clusters gets shock heated and shock fronts develop. At a later stage (right), the cluster cores merge and can develop additional shock fronts, while the initial shock waves travel outwards.
Feretti et al. (2001) classified the radio feature in the NE as a radio relic, which spatially coincides with the position of the NE shock front. The authors also discuss the possibility that this radio feature is part of a wide-angle-tailed (WAT) radio source with a size of $\sim 1.3 \mathrm{Mpc}$, which, however, seems unlikely as these features are usually smaller and located in the central parts of clusters. Additionally, Feretti et al. (2004) measured the spectral index map of A2163 and found that the index flattens at the peak position of the NE relic and steepens towards the edges. A flatter electron spectrum points towards a more energetic particle population. Interestingly, the spectral index map also shows an elongated region of a flatter spectral index located close to the outer SW shock front, however, this feature has not (yet) been classified as a radio relic but seems likely to be related to the shock front.

In common understanding, the highly energetic electrons produced in merger shocks are responsible for radio emission and, in particular, can form radio relics. This was also seen, for example, by Macario et al. (2011) and Akamatsu et al. (2012, 2015) for the disturbed clusters A754, A3376, and CIZA $\mathrm{J} 2242.8+5301$, which all exhibit radio relics that spatially coincide with the position of X-ray detected shock fronts.

The clumpy shape of the spectral index map of A2163, as discussed in Feretti et al. (2004), and the flatter shape of the spectra close to the shock positions suggests a reacceleration of the electron population at sites of recent merger activity. Feretti et al. (2004) found that in relatively undisturbed cluster regions, the index steepens with distance from the cluster center. For the interpretation of this steepening, the authors refer to Brunetti et al. (2001), who observed this behavior for the Coma galaxy cluster and explained it with a two-phase model, where in the first phase an energetic electron population is continuously injected by shocks, AGN activity, and/or turbulences and later, in the second phase, reaccelerated, for example by recent merging activity.

Comparison of the X-ray shock positions to the findings obtained by Bourdin et al. (2011) with XMM-Newton and Chandra shows that the inner shock in the SW direction is located close in front of a cool spot as depicted in Fig. 6. In the scenario suggested by Bourdin et al. (2011) and Maurogordato et al. (2008), the merger in A2163 took place in the E-W direction (projected onto the plane of the sky) and the cold spot is likely a cool core relic of one of the merging constituents, which is moving westwards. This scenario is supported by our findings as it seems likely that the inner SW shock front is related to the cool core. Also a cold front was identified previously in a sector at the north-west boundary of the cold spot using Chandra data (Bourdin et al. 2011), however, it cannot be resolved with Suzaku.

The presence of multiple shock fronts with somewhat different Mach numbers and shock velocities suggests a rather complex merger scenario. However, a simplified possible scenario is depicted in Fig. 7, which was initially suggested by Markevitch et al. (2000) for cluster A2142, but is also conceivable for A2163. Two merging constituents approach each other and develop multiple shock fronts in the ICM, which travel outwards. In an ideally symmetric head-on collision with equal masses, the velocities of these shock fronts are similar. The cores of the clusters merge and ahead of these dense (and often cool) structures, additional shock fronts can occur. This is similar to our findings for A2163, where the inner shock front in the SW direction is situated close in front of the moving cool core and two shock fronts are traveling outwards at larger radii in both directions. We find the two outer shocks at roughly equal distance from the X-ray peak. However, the Mach numbers and velocities are quite different, which is likely to be due to deviations from an ideal head-on collision and/or unequal merging constituents. Projection effects can also play a role as it is difficult to constrain the angle between the line of sight and the merging axis, which can even lead to an underestimation of the Mach number (e.g., Skillman et al. 2013; Hong et al. 2015). Additionally, the abovedescribed scenario is highly idealized and in real situations, turbulent gas motions as well as multiple minor mergers may occur. This is supported by the previously mentioned radio and optical studies, which identified several substructures in A2163. Furthermore, in the course of the merging process of the dense cores, the position of the X-ray peak can change and does not necessarily coincide with the origin of the initial shock waves, which might contribute to the fact that the two shock fronts lie at roughly the same distance from the X-ray peak despite their different velocities.

\section{Summary}

We identified three shock fronts in A2163 using Suzaku data in two azimuthal directions and compared the X-ray findings to the radio morphology, obtained from archival VLA observations at $20 \mathrm{~cm}$. The NE direction exhibits one shock at a distance of $1.4 \mathrm{Mpc}$ from the center, with a typical Mach number for X-ray detected shocks of $M=1.7_{-0.2}^{+0.3}$, estimated from the discontinuity in the temperature profile. The SW direction shows a more complicated temperature profile and we identify two shocks. One is located at $0.7 \mathrm{Mpc}$ with $M=1.5_{-0.3}^{+0.5}$, estimated as the 
average from the density and temperature discontinuities; the other one lies at $1.3 \mathrm{Mpc}$ and is one of the strongest detected shocks with $M=3.2_{-0.7}^{+0.6}$, estimated from the temperature jump. Previous studies in the radio and optical regime identified several substructures, which hint at a complicated merger scenario. The radio contours match the positions of the X-ray detected shocks well. The NE shock front coincides with the position of a radio relic, which was identified previously by Feretti et al. (2001, 2004). Their radio spectral index map also shows a region of flatter index close to the outer SW shock front, which hints at an electron reacceleration mechanism at sites of ongoing merging activity. Close to the inner shock front in the SW direction, Bourdin et al. (2011) identified a moving cool core remnant, which suggests a connection between the two. The merging scenario can qualitatively be explained by two merging constituents, which passed each other and developed shock fronts at an early stage of the merger. These shock fronts traveled outwards, while the dense cluster cores merged and formed additional shock and cold fronts ahead of them. The different Mach numbers and shock velocities, as well as the substructures identified in the radio and optical regime, suggest that the real merger situation is more complex and is likely to have occurred off-axis with unequal merging constituents.

Acknowledgements. ST acknowledges support from the Bonn-Cologne Graduate School of Physics and Astronomy and the Argelander-Institut für Astronomie. THR acknowledges support from the German Aerospace Agency (DLR) with funds from the Ministry of Economy and Technology (BMWi) through grant 50 OR 1514. MWS acknowledges support for this work from Transregio Programme TR33 of the German Research Foundation (Deutsche Forschungsgemeinschaft). This work was supported in part by JSPS KAKENHI Grant Number 16K05295 (NO).

\section{References}

Akamatsu, H., Hoshino, A., Ishisaki, Y., et al. 2011, PASJ, 63, 1019 Akamatsu, H., Takizawa, M., Nakazawa, K., et al. 2012, PASJ, 64, 67

Akamatsu, H., van Weeren, R. J., Ogrean, G. A., et al. 2015, A\&A, 582, A87

Akamatsu, H., Mizuno, M., Ota, N., et al. 2017, A\&A, 600, A100

Angus, G. W., \& McGaugh, S. S. 2008, MNRAS, 383, 417

Asplund, M., Grevesse, N., Sauval, A. J., \& Scott, P. 2009, ARA\&A, 47, 481

Borgani, S., \& Guzzo, L. 2001, Nature, 409, 39

Botteon, A., Gastaldello, F., \& Brunetti, G. 2018, MNRAS, 476, 5591

Bourdin, H., Arnaud, M., Mazzotta, P., et al. 2011, A\&A, 527, A21

Bourdin, H., Mazzotta, P., Markevitch, M., Giacintucci, S., \& Brunetti, G. 2013, ApJ, 764, 82

Brunetti, G., \& Lazarian, A. 2007, MNRAS, 378, 245

Brunetti, G., \& Lazarian, A. 2011, MNRAS, 412, 817

Brunetti, G., Setti, G., Feretti, L., \& Giovannini, G. 2001, MNRAS, 320, 365

De Luca, A., \& Molendi, S. 2004, A\&A, 419, 837

Eckert, D., Gaspari, M., Vazza, F., et al. 2017, ApJ, 843, L29

Elbaz, D., Arnaud, M., \& Boehringer, H. 1995, A\&A, 293, 337

Ettori, S., Fabian, A. C., Allen, S. W., \& Johnstone, R. M. 2002, MNRAS, 331, 635

Feretti, L., Fusco-Femiano, R., Giovannini, G., \& Govoni, F. 2001, A\&A, 373, 106

Feretti, L., Orrù, E., Brunetti, G., et al. 2004, A\&A, 423, 111

Ghizzardi, S., Rossetti, M., \& Molendi, S. 2010, A\&A, 516, A32

Ha, J.-H., Ryu, D., \& Kang, H. 2018, ApJ, 857, 26

Hattori, S., Ota, N., Zhang, Y.-Y., Akamatsu, H., \& Finoguenov, A. 2017, PASJ, 69,39

Hayashi, E., \& White, S. D. M. 2006, MNRAS, 370, L38

Hong, S. E., Kang, H., \& Ryu, D. 2015, ApJ, 812, 49

Hoshino, A., Henry, J. P., Sato, K., et al. 2010, PASJ, 62, 371

Lee, J., \& Komatsu, E. 2010, ApJ, 718, 60

Macario, G., Markevitch, M., Giacintucci, S., et al. 2011, ApJ, 728, 82

Markevitch, M., \& Vikhlinin, A. 2001, ApJ, 563, 95
Markevitch, M., \& Vikhlinin, A. 2007, Phys. Rep., 443, 1

Markevitch, M., Ponman, T. J., Nulsen, P. E. J., et al. 2000, ApJ, 541, 542

Markevitch, M., Gonzalez, A. H., David, L., et al. 2002, ApJ, 567, L27

Maurogordato, S., Cappi, A., Ferrari, C., et al. 2008, A\&A, 481, 593

McCarthy, I. G., Bower, R. G., Balogh, M. L., et al. 2007, MNRAS, 376, 497

McMullin, J. P., Waters, B., Schiebel, D., Young, W., \& Golap, K. 2007, ASP Conf. Ser., 376, 127

Merloni, A., Predehl, P., \& Becker, W. et al. 2012, ArXiv e-prints [arXiv:1209.3114]

Ogrean, G. A., Brüggen, M., van Weeren, R., et al. 2014, MNRAS, 440, 3416

Okabe, N., Bourdin, H., Mazzotta, P., \& Maurogordato, S. 2011, ApJ, 741, 116

Ota, N., Nagayoshi, K., Pratt, G. W., et al. 2014, A\&A, 562, A60

Perley, R. A., \& Butler, B. J. 2013, ApJS, 204, 19

Radovich, M., Puddu, E., Romano, A., Grado, A., \& Getman, F. 2008, A\&A, 487, 55

Rau, U., \& Cornwell, T. J. 2011, A\&A, 532, A71

Rosen, S. R., Webb, N. A., Watson, M. G., et al. 2016, A\&A, 590, A1

Simionescu, A., Roediger, E., Nulsen, P. E. J., et al. 2009, A\&A, 495, 721

Skillman, S. W., Xu, H., Hallman, E. J., et al. 2013, ApJ, 765, 21

Springel, V., \& Farrar, G. R. 2007, MNRAS, 380, 911

Springel, V., White, S. D. M., Jenkins, A., et al. 2005, Nature, 435, 629

Struble, M. F., \& Rood, H. J. 1999, ApJS, 125, 35

Thölken, S., Lovisari, L., Reiprich, T. H., \& Hasenbusch, J. 2016, A\&A, 592, A37

Vogelsberger, M., Genel, S., Springel, V., et al. 2014, MNRAS, 444, 1518

Willingale, R., Starling, R. L. C., Beardmore, A. P., Tanvir, N. R., \& O'Brien, P. T. 2013, MNRAS, 431, 394

\section{Appendix A: Additional tables}

Table A.1. Fit results in the NE direction.

\begin{tabular}{c|c|c|c}
\hline \hline Annulus (arcmin) & $T(\mathrm{keV})$ & $Z\left(Z_{\odot}\right)$ & norm $^{a}$ \\
\hline $0-1$ & $14.13 \pm 1.65$ & $0.68 \pm 0.17$ & $76.53 \pm 0.94$ \\
\hline $1-2$ & $17.01 \pm 1.96$ & $0.33 \pm 0.11$ & $151.91 \pm 0.98$ \\
$2-3$ & $16.03 \pm 2.89$ & & $75.36 \pm 0.50$ \\
\hline $3-4$ & $17.87 \pm 5.12$ & & $43.37 \pm 0.40$ \\
$4-5$ & $14.88 \pm 2.46$ & $0.60 \pm 0.15$ & $35.70 \pm 0.45$ \\
$5-6$ & $19.16 \pm 5.23$ & & $23.08 \pm 0.37$ \\
\hline $6-7$ & $10.38 \pm 2.13$ & & $20.34 \pm 0.36$ \\
$7-8$ & $10.71 \pm 5.00$ & $0.20 \pm 0.16$ & $15.16 \pm 0.39$ \\
$8-9$ & $13.33 \pm 6.74$ & & $9.81 \pm 0.35$ \\
$9-10$ & $9.76 \pm 4.24$ & & $6.03 \pm 0.32$ \\
\hline
\end{tabular}

Notes. ${ }^{(a)}$ Normalization in the full annulus: norm = $\frac{10^{-18}}{4 \pi\left[D_{\mathrm{A}}(1+z)\right]^{2}} \int n_{\mathrm{e}} n_{\mathrm{H}} \mathrm{d} V \mathrm{~cm}^{-5}$ with $D_{\mathrm{A}}$ being the angular diameter distance to the source.

Table A.2. Fit results in the SW direction.

\begin{tabular}{c|c|c|c}
\hline \hline Annulus (arcmin) & $T(\mathrm{keV})$ & $Z\left(Z_{\odot}\right)$ & norm $^{a}$ \\
\hline $0-1$ & $14.21 \pm 1.25$ & $0.57 \pm 0.11$ & $84.26 \pm 0.48$ \\
\hline $1-2$ & $15.01 \pm 1.46$ & $0.31 \pm 0.07$ & $149.22 \pm 0.52$ \\
$2-3$ & $15.53 \pm 1.32$ & & $64.80 \pm 0.30$ \\
\hline $3-4$ & $12.09 \pm 1.42$ & & $22.91 \pm 0.19$ \\
$4-5$ & $13.59 \pm 2.70$ & $0.12 \pm 0.12$ & $11.84 \pm 0.17$ \\
$5-6$ & $16.84 \pm 3.94$ & & $7.98 \pm 0.17$ \\
\hline $6-7$ & $8.08 \pm 1.21$ & & $6.03 \pm 0.19$ \\
$7-8$ & $7.41 \pm 1.55$ & $0.31 \pm 0.12$ & $4.36 \pm 0.20$ \\
$8-9$ & $4.73 \pm 1.12$ & & $2.87 \pm 0.20$ \\
\hline
\end{tabular}

Notes. ${ }^{(a)}$ Same definition as in Table A.1. 